\title{
Implantation et évaluation d'un programme de formation sur la lecture interactive destiné à des parents d'enfants de maternelle 5 ans
}

\author{
MONICA BOUDREAU \\ Université du Québec à Rimouski/campus Lévis \\ ISABELLE BEAUDOIN \\ Université du Québec à Rimouski/campus Lévis \\ JULIE MÉLANÇON \\ Université du Québec à Rimouski/campus Lévis
}

Résumé

Cet article présente une recherche quasi expérimentale qui avait pour but d'implanter et d'évaluer, auprès de 59 parents d'enfants de maternelle 5 ans du Québec, un programme de formation sur l'exploitation de la littérature jeunesse pour favoriser, entre autres, le développement langagier des enfants. Le programme, intitulé Les mille et un plaisirs de lire avec toi, visait à encourager les parents à fournir un soutien de qualité à leur enfant lors de la lecture interactive d'albums. Afin d'assurer ce soutien, des ateliers de formation leur ont été offerts et des trousses littéraires ont circulé dans les familles pendant six mois. Cet article décrit la recherche à laquelle ont pris part les parents et présente les résultats qui révèlent que le programme a contribué à les sensibiliser à l'importance de la lecture interactive et à celle de susciter des interactions de qualité lors de l'animation de ce type d'activité.

\section{Mots-clés}

lecture interactive, compétences langagières, interventions parentales, littératie familiale, éducation préscolaire

\section{Introduction}

Plusieurs recherches menées au Québec font état d'un écart entre les compétences langagières des enfants d'âge préscolaire. Il est reconnu que certains enfants ont moins d'occasions de vivre des expériences familiales en lien avec la lecture et l'écriture, dont ceux issus de milieux défavorisés (Simard, Tremblay, Lavoie \& Audet, 2013; Poissant \& Garnache, 2016). Compte tenu des besoins de ces enfants plus vulnérables et considérant les bénéfices de la lecture interactive d'albums de littérature jeunesse (Kispal, 2008; Makdissi, Boisclair \& Sirois, 2010), la présente recherche a mis en place un programme de formation proposé à dix enseignantes de maternelle 5 ans œuvrant dans des écoles de milieux défavorisés d'une commission scolaire francophone de la région de Québec ainsi qu'aux parents de 115 enfants de ces classes. Le programme, intitulé Les mille et un plaisirs de lire avec toi, misait sur la littératie en milieu scolaire tout en favorisant une collaboration école-famille. Il était composé de deux volets. Le premier, destiné aux enseignantes du groupe expérimental (GE), comprenait trois ateliers de formation portant sur les types d'ouvrages à exploiter en classe et l'importance de la lecture interactive, notamment pour le développement des compétences langagières des enfants. Le deuxième volet assurait le 
lien entre l'école et la famille par l'entremise de trousses littéraires qui circulaient dans les maisons et de trois ateliers de formation offerts aux parents du GE à propos des bénéfices de la lecture interactive pour soutenir ces compétences.

Le présent article portera spécifiquement sur ce deuxième volet de la recherche. Bien que l'étude ait considéré à la fois les facteurs éducatifs, familiaux et ceux reliés à l'enfant, nous nous attarderons ici essentiellement au volet concernant les parents.

\section{Problématique}

La stimulation du langage oral et écrit est un enjeu incontournable pour la réussite éducative des enfants d'âge préscolaire (Conseil supérieur de l'éducation [CSÉ], 2012). Cependant, des recherches montrent clairement que les enfants n'ont pas tous le même niveau de préparation lorsqu'ils arrivent à la maternelle (Britto \& Brooks-Gunn, 2001; Morris et al., 2008; Simard et al., 2013). Les enfants issus de milieux défavorisés sont souvent les plus désavantagés (Boivin et al., 2012; Poissant \& Garnache, 2016). La situation de ces enfants est préoccupante puisqu'ils vivent peu d'expériences en lien avec la lecture et l'écriture, ont des connaissances plus faibles en émergence de l'écrit et présentent des écarts importants en ce qui a trait à l'étendue de leur vocabulaire (Hart \& Risley, 2003; Desrosiers \& Ducharme, 2006). Il est reconnu que les interventions préventives en petite enfance peuvent modifier le parcours de vie de ces enfants issus de familles défavorisées (Irwin, Siddiqi \& Hertzman, 2007; Domitrovich et al., 2010; Duncan \& Sojourner, 2013). Les recommandations issues de Janosz et ses collaborateurs (2013) rappellent l'importance d'intervenir tôt (Duncan \& Magnuson, 2013; Marmot et al., 2010) pour prévenir les risques de décrochage scolaire, en soutenant notamment l'apprentissage de la lecture.

Afin de mettre en place des actions efficaces auprès des jeunes enfants, qu'ils soient issus ou non de milieux défavorisés, il apparait important de s'intéresser aux parents qui sont les premiers éducateurs de leur enfant. Des recherches ont montré que des programmes de littératie familiale axés sur la littérature jeunesse peuvent avoir des effets positifs sur la qualité des interventions des parents (Beauregard, Carrignan \& Létourneau, 2011; Baker, 2013; Boudreau, 2017) et que la lecture interactive d'albums de littérature jeunesse s'avère un contexte à privilégier pour soutenir le développement du langage oral et écrit du jeune enfant (Kispal, 2008; Makdissi et al., 2010; Boudreau \& Grondin, 2012, Dionne, 2013; Montesinos-Gelet, Dupin de Saint-André \& Bourdeau, 2015; Saracho, 2017).

En somme, puisqu'il importe d'intervenir tôt pour favoriser la réussite éducative, l'éducation préscolaire doit jouer un rôle de premier plan dans le développement des enfants, particulièrement de ceux issus de milieux défavorisés (Irwin et al., 2007). Or, tel que le rappellent Myre-Bisaillon et ses collaborateurs (2014), les actions déployées s'attardent souvent à l'intervention directe auprès de l'enfant d'âge préscolaire plutôt qu'à la formation des acteurs qui interviennent auprès de lui, notamment ses parents. En ce sens, la présente étude se distingue par son souci de mieux outiller les personnes jouant un rôle de premier plan dans la vie de l'enfant, soit ses parents. De plus, le choix de leur offrir une formation visant le développement du langage oral et écrit à partir de l'exploitation d'albums de littérature jeunesse s'avère particulièrement pertinent, notamment en milieux défavorisés où l'on risque de retrouver davantage d'enfants d'âge préscolaire présentant des défis sur le plan du développement de ces compétences pourtant fortement reliées à leur réussite ultérieure en lecture et en écriture (Pagani, Fitzpatrick, Belleau \& Janosz, 
2011; Simard et al., 2013; Myre-Bisaillon et al., 2014; Puentes-Neuman, Myre-Bisaillon, Collette, Larose \& Morin, 2015). Le programme proposé, s'il génère l'effet attendu, devrait ainsi contribuer à réduire l'impact négatif des facteurs de risque inhérents au niveau de défavorisation du milieu.

\section{Cadre théorique}

Le vocabulaire, la conscience phonologique et les lettres de l'alphabet sont des facteurs de protection largement reconnus pour la réussite en lecture et en écriture (Ehri, Nunes, Stahl \& Willows, 2001; Foulin \& Pacton, 2006; Sénéchal \& LeFevre, 2002). Or, au moment de l'entrée à la maternelle, un enfant sur trois de milieux défavorisés arrive vulnérable, notamment dans la sphère langagière, et près d'un enfant de maternelle sur dix montre un retard persistant quant à la compréhension de mots lorsque comparé à des pairs du même âge (Desrosiers \& Ducharme, 2006). Dès l'âge de trois ans, il existe un écart de 600 mots connus entre les enfants de milieux favorisés et ceux de milieux défavorisés (Hart \& Risley, 2003). Ces constats sont inquiétants puisque le développement du langage oral est un facteur central pour l'acquisition du langage écrit (Wasik, Hindman \& Snell, 2016). Ainsi, plusieurs chercheurs ont maintes fois relevé l'importance de faire vivre à tous les enfants des expériences variées et riches pour soutenir le développement de leur vocabulaire, de leur conscience phonologique et de leur connaissance des lettres de l'alphabet avant qu'ils n'entrent en première année du primaire (Boudreau, Saint-Laurent \& Giasson, 2009; Brodeur, Gosselin, Mercier, Legault \& Vanier, 2006; Cantin, Bouchard \& Charron, 2010; Fayol, 2017; Foulin \& Pacton, 2006), et ce, grâce à des contacts soutenus avec la littérature jeunesse (Montesinos-Gelet et al., 2015).

\section{Facteurs familiaux}

Il est maintenant bien reconnu dans la littérature scientifique que les parents jouent un rôle de premier plan dans le développement des compétences langagières de leur enfant (Boudreau et al., 2009; Britto \& Brooks-Gunn, 2001; Sénéchal, 2008). En effet, les interactions quotidiennes entre le parent et son enfant en lien avec l'éveil à la lecture et à l'écriture permettent de sensibiliser ce dernier au langage oral et écrit (Beauregard et al., 2011; Boudreau \& Grondin, 2012; Charron, Gagnon \& Fortin-Clément, 2013; Sénéchal \& LeFevre, 2002) et les activités de littératie familiale se rapprochant de celles vécues en milieu éducatif ont un effet positif sur l'apprentissage de la lecture de l'enfant (Jung, 2016; Maltais, 2007; ministère de l'Éducation du Québec [MEQ], 2003; Ziv, Smadja \& Aràm, 2014). Tel que le rapportent Myre-Bisaillon et ses collaborateurs (2010), la majorité des parents questionnés dans leur étude affirment être peu sensibilisés et formés à réaliser des activités d'éveil à la lecture et à l'écriture. Ce manque de connaissances des parents affaiblit l'impact de leurs interventions (Aràm, Fine \& Ziv, 2013; Boisvert \& Gagnon, 2005), et ce, particulièrement en milieux défavorisés où les parents manifestent un plus grand besoin de rencontres de formation pour accompagner leur enfant (Deslandes \& Bertrand, 2001). De surcroit, la lecture d'histoires ne semble pas une pratique fréquente; Lavoie et Fontaine (2016) ont observé que $24 \%$ des parents interrogés déclarent lire une seule et parfois même aucune histoire par semaine à leur enfant. Or, la qualité des interactions parent-enfant durant la lecture est l'un des aspects au cœur du développement du langage oral et écrit (Dionne, 2013; Mol, Bus \& de Jong, 2009; Saracho, 2017). Ainsi, les mesures préventives devraient outiller les parents à offrir des activités qui favorisent l'apprentissage de la lecture 
et de l'écriture (Tamis-LeMonda \& Rodriguez, 2014). Tel que le rappellent Taylor, Zubrick et Christensen (2016, p. 296), «[1]a lecture de livres est l'un des plus importants investissements que peuvent faire les parents pour le développement de la littératie de leurs enfants ».

\section{Accompagnement et formation des parents}

Plusieurs chercheurs et intervenants font la promotion de programmes de littératie familiale destinés à des parents d'enfants d'âge préscolaire puisque, comme le souligne Masny (2008), ils permettent de briser le cercle vicieux de l'échec scolaire. Charron et ses collaboratrices (2013), Boudreau (2017) et Giasson et Saint-Laurent (2004) suggèrent de mettre en place des ateliers destinés aux parents afin de les informer des différentes façons d'aider et de soutenir leur enfant à la maison. En effet, souvent intuitives, les interventions des parents gagnent à être enrichies et soutenues par un accompagnement explicite (Baker, 2013; Ziv et al., 2014). Pour que ces programmes soient plus efficaces, Beauregard et ses collaboratrices (2011) recommandent de bien préparer les parents : leur expliquer les buts et objectifs du programme, insister sur l'importance de réaliser les activités dans le plaisir, leur donner la parole, tenir compte de la disponibilité des familles, etc.

Les résultats de plusieurs programmes de littératie familiale mis en place auprès de parents d'enfant de maternelle (Beauregard et al., 2011; Boudreau, 2017; Myre-Bisaillon et al., 2014) indiquent des changements dans leurs pratiques de litttératie familiale après leur participation à de tels programmes. Ainsi, sachant que l'intervention éducative trouve d'abord sa source dans les familles, que le fait de former les parents a un effet positif sur le rendement en lecture de leur enfant (Aràm et al., 2013; Sénéchal, 2008) et que peu de formations et d'accompagnement sont réalisés directement auprès des familles (MyreBisaillon et al., 2014), nous devons socialement chercher des moyens de les soutenir afin qu'elles deviennent plus compétentes pour aider leur enfant et plus assidues dans leur démarche éducative (Britto \& Brooks-Gunn, 2001; Thériault \& Lavoie, 2004).

\section{Lecture interactive d'albums de littérature jeunesse}

On reconnait aujourd'hui que la lecture interactive d'albums de littérature jeunesse met en place une réelle zone d'intersubjectivité littéraire où le dialogue permet de construire une interprétation valable du texte (Makdissi et al., 2010), suscitant des interactions parent-enfant de qualité, c'est-à-dire axées sur des informations allant au-delà de ce qui se trouve explicitement véhiculé par le texte et par l'image (ex. : prédictions, inférences, clarifications, rappels, etc.). Le dialogue favorisé par des interventions avant, pendant et après la lecture s'avère particulièrement bénéfique pour le développement du vocabulaire (Godin, Godard, Chapleau \& Gagné, 2015; Marulis \& Neuman, 2010; Wasik et al., 2016) et de la syntaxe (Blachowicz \& Fisher, 2015), la compréhension en lecture (Dougherty Stahl, 2014; Dupin de Saint-André, Montésinos-Gelet \& Morin, 2012;), la compréhension de nouveaux concepts (Zucker, Justice, Piasta \& Kaderavek, 2010) et l'émergence de l'écrit (la conscience de l'écrit et de ses fonctions, des concepts, des conventions et des caractéristiques de la langue écrite ainsi que des relations entre l'oral et l'écrit) (Swanson et al., 2011).

Puisque l'apprentissage de la lecture et de l'écriture pour un enfant commence bien avant l'entrée à l'école, il devient important de s'intéresser aux parents qui sont les personnes pouvant amorcer l'aventure de la lecture et de l'écriture auprès de leur enfant 
(Thériault \& Lavoie, 2004). Socialement, l'intérêt de chercher des moyens de les aider et de les soutenir afin qu'ils deviennent plus compétents pour accompagner leur enfant ne fait pas de doute. En effet, il apparait primordial de leur faire prendre conscience qu'ils peuvent intervenir à une période sensible du développement de leur enfant et ainsi empêcher que des problèmes scolaires ne prennent des proportions importantes (Beauregard et al., 2011; Boudreau \& Grondin, 2012; Sénéchal, 2008).

\section{Question et objectifs de recherche}

La recherche dont il est question dans le présent article s'inscrit dans une programmation de recherche plus vaste qui proposait d'étudier l'effet d'un programme de formation sur l'exploitation de la littérature jeunesse auprès de parents et d'enseignantes pour soutenir le développement des compétences langagières et sociales d'enfants de maternelle 5 ans. Le programme Les mille et un plaisirs de lire avec toi ne visait pas à scolariser l'enfant mais plutôt à accompagner les parents et les enseignantes dans l'exploitation des livres de littérature jeunesse dans des situations signifiantes du quotidien qui respectent les besoins et le développement de l'enfant. Plus précisément, la recherche souhaitait répondre à la question suivante : Quel est l'effet du programme sur la qualité des interventions effectuées par les parents et les enseignantes pour soutenir les compétences langagières et sociales des enfants lors de la lecture interactive d'albums de littérature jeunesse?

Nous rappelons que cet article porte uniquement sur le volet traitant de la qualité des interventions des parents lors de la lecture interactive d'albums de littérature jeunesse pour soutenir les compétences langagières de leur enfant. Il s'intéresse donc aux deux objectifs spécifiques suivants : 1) implanter le programme et accompagner les parents dans leurs interventions entourant l'exploitation de la littérature jeunesse et 2) évaluer l'effet du programme sur la qualité des interventions réalisées par les parents lors de la lecture interactive d'albums pour favoriser le développement des compétences langagières de leur enfant.

\section{Méthodologie}

Cette section présente la méthodologie qui a été retenue afin de répondre aux deux objectifs poursuivis par la recherche.

\section{Participants}

Afin de répondre aux objectifs visés, un devis quasi expérimental a été retenu. Il visait à comparer deux groupes : un groupe expérimental (GE) soumis au programme proposé et un groupe témoin (GT) n'en ayant pas bénéficié. Au total, 115 dyades parentenfant de maternelle 5 ans d'écoles de milieux urbain et rural d'une commission scolaire francophone de la région de Québec ont participé à l'étude, soit 59 dyades (2 écoles) dans le $\mathrm{GE}^{1}$ ainsi que 56 dyades (3 écoles) dans le GT. L'une des écoles du GE provient d'un milieu urbain et l'autre d'un milieu rural. Quant aux écoles du GT, deux proviennent d'un

\footnotetext{
${ }^{1}$ Les parents ont été recrutés sans exercer de contrôle sur la proportion des mères/pères. Au premier atelier, 48 mères et dix pères étaient présents. Bien que les données ne soient pas disponibles pour les ateliers 2 et 3 , les chercheuses ont noté un haut taux de participation de la part des pères dans les différents milieux. Lors de la complétion du questionnaire, ce sont majoritairement les mères (48 GE, $50 \mathrm{GT}$ ) qui ont répondu au questionnaire autodéclaré pour les deux temps de mesure.
} 
milieu urbain et une d'un milieu rural. Les parents participant ont tous reçus un formulaire les invitant à exprimer par écrit leur consentement à l'égard de leur participation et de celle de leur enfant. Ce sont les conseillères pédagogiques œuvrant à l'éducation préscolaire qui ont sélectionné les écoles participantes, et ce, afin de répondre aux besoins de leur milieu. Ces écoles présentent un indice du seuil de faible revenu (SFR) variant entre 1 et 8 et un indice de milieu socioéconomique (IMSE) variant entre 2 et 7 (Ministère de l'Éducation et de l'Enseignement supérieur [MEES], 2015). ${ }^{2}$ Les données sociodémographiques recueillies indiquent que $53,3 \%$ des mères et $71,7 \%$ des pères du GE et $43,7 \%$ des mères et $79,6 \%$ des pères du GT n'ont pas de diplôme universitaire.

\section{Questionnaire sur les pratiques reliées à la lecture interactive d'albums de littérature jeunesse}

Les parents des GE et GT ont répondu, en novembre 2015, à un questionnaire autodéclaré les invitant à estimer, sur une échelle de type Likert à quatre niveaux ( 0 - jamais ou presque jamais, 1-à l'occasion, 2- souvent et 3-très souvent ou toujours), la fréquence de leur recours à 16 interventions de qualité pouvant être réalisées avant, pendant et après la lecture interactive d'albums pour soutenir le développement langagier de leur enfant (ex. : Lorsque vous lisez un livre à votre enfant, - vous vous attardez à la page couverture pour discuter avec lui de quoi parlera le livre, - vous prenez le temps de discuter avec lui des illustrations du livre, - vous lui demandez de prédire ce qui va arriver par la suite dans 1'histoire, - vous attirez son attention sur les mots qui riment, etc.). Ce questionnaire a été élaboré par les chercheuses à partir des postulats théoriques concernant les interventions de qualité pouvant être soulevées lors de la lecture (Aràm et al., 2013; Boudreau, 2005; Dionne, 2013; Giasson, 1995). Une première version du questionnaire a été préexpérimentée par cinq parents d'enfant de maternelle 5 ans, ce qui a permis de clarifier certains énoncés. La validité de contenu a ensuite été réalisée par deux expertes en lecture interactive d'albums. La consistance interne du questionnaire, calculée auprès de 118 parents, est excellente (alpha de Cronbach $=90$ ). L'indice de stabilité test-retest mesuré auprès des 59 parents du GT est acceptable $(r=, 72)$. De plus, l'analyse factorielle confirme l'unidimensionnalité de l'échelle : la première valeur propre est de 6,48 et explique $40,48 \%$ de la variance alors que les suivantes, toutes inférieures à 1,37, expliquent chacune 8,5\% ou moins de la variance $(n=118)$. Des questions portant sur des renseignements sociodémographiques tels que le niveau d'études le plus élevé des deux parents et le revenu familial ont également été posées aux participants. Enfin, des questions visant à recueillir des données préliminaires sur les habitudes de lecture à la maison ont permis de vérifier si les parents faisaient la lecture à leur enfant ${ }^{3}$.

En juin 2016, les parents du GE et du GT ont été invités à répondre à nouveau au questionnaire. Ils ont reçu comme consigne de le faire remplir par le même parent que celui qui avait répondu au questionnaire la première fois. Afin de connaitre leur appréciation concernant leur participation au programme, six questions ont été ajoutées au questionnaire destiné aux parents du GE (ex. : - leur appréciation de l'expérience, - l'utilité des formations, - l'impact du projet sur la manière dont ils lisent à leur enfant, etc.).

\footnotetext{
${ }^{2}$ Les conseillères pédagogiques ont considéré à la fois les deux indices du MEES (2015) pour identifier les écoles retenues. Ainsi, deux profils de milieux ont été identifiés : un milieu rural présentant un ISR élevé, mais un IMSE faible, ainsi qu'un milieu urbain présentant un ISR faible, mais un IMSE élevé.

${ }^{3}$ Notons que tous les parents sondés ont déclaré faire la lecture à leur enfant.
} 


\section{Expérimentation}

Deux stratégies ont été mises en place dans le cadre du programme Les mille et un plaisirs de lire avec toi pour permettre aux parents du GE de soutenir leur enfant dans le développement de leurs compétences langagières : des ateliers de formation et des trousses littéraires, élaborés à partir de recommandations issues de la littérature scientifique, leur ont été offerts.

\section{Ateliers de formation destinés aux parents $d u G E$}

Les parents du GE ont bénéficié de trois ateliers de formation de 90 minutes (novembre 2015, février et avril 2016) offerts par l'équipe de recherche. Ces ateliers, qui se déroulaient en soirée dans les établissements scolaires fréquentés par les enfants, visaient à leur faire part de l'importance des compétences langagières et sociales pour la réussite éducative de leur enfant et à leur présenter des moyens concrets pour favoriser leur développement à l'aide d'albums de littérature jeunesse.

Atelier 1 : présentation du programme Les mille et un plaisirs de lire avec toi et des trousses littéraires

Cet atelier était l'occasion de présenter l'équipe de recherche et les objectifs poursuivis par le programme auquel ils avaient accepté de participer. Après avoir rempli le questionnaire sur leurs pratiques reliées à la lecture interactive d'albums de littérature jeunesse à leur enfant (prétest), les parents ont bénéficié d'explications sur les trousses littéraires et leur fonctionnement. L'équipe a insisté sur l'aspect ludique de la démarche et sur l'importance de vivre une expérience agréable en lien avec la lecture. Les parents ont eu l'occasion de manipuler les trousses et de se familiariser avec leur contenu.

Atelier 2 : la lecture interactive au quotidien

Cet atelier portait sur la lecture interactive d'albums de littérature de jeunesse, et plus spécifiquement sur les interventions à réaliser avant, pendant et après la lecture. La formation a débuté par la modélisation d'une lecture interactive à haute voix exécutée par une assistante de recherche. Pour l'occasion, les enfants ont été invités à accompagner leur parent à l'école. Ils étaient réunis au tapis de rassemblement de la classe, devant l'assistante de recherche, tandis que les parents, à l'écart, assistaient à la modélisation. L'ouvrage utilisé était l'album d'histoire $L e$ jeune loup qui n'avait pas de nom (Mourlevat \& Bénazet, 1998). Cet album a été choisi en raison des multiples occasions d'interactions que son récit offrait (Dupin de Saint-André, 2011). Une fois la lecture interactive terminée, les enfants ont accompagné l'assistante dans un autre local, afin de permettre à l'équipe de recherche d'effectuer un retour avec les parents sur la modélisation de lecture interactive à laquelle ils venaient d'assister, principalement sur les interventions avant, pendant et après la lecture. Un livret rappelant aux parents les pratiques à privilégier à chacune des étapes de la lecture leur a aussi été remis. De plus, ces derniers ont été invités à s'exprimer quant à la gestion des trousses qu'ils recevaient hebdomadairement depuis le mois de novembre et à partager leurs astuces et leurs 
réussites à cet égard. L'atelier s'est terminé par une activité de lecture parentenfant.

Atelier 3 : les compétences sociales et la littérature jeunesse

Le troisième atelier a repris la même formule, mais cette fois avec l'objectif de montrer aux parents comment, à partir d'un album de littérature jeunesse, il était possible d'amener l'enfant à réfléchir à propos des émotions et de la résolution de conflits. L'ouvrage utilisé était l'album d'histoire Sans toi! (Côté, 2011).

\section{Trousses littéraires destinés aux parents du GE}

Au total, 18 trousses littéraires ont été élaborées par l'équipe de recherche et ont circulé dans les milieux familiaux de novembre 2015 à mai 2016. Les trousses comprenaient des types d'ouvrage variés tels que des albums d'histoire, des albums documentaires, des abécédaires ou des livres-jeux. En plus de l'album sélectionné, on retrouvait dans chaque trousse un livret présentant les interventions à effectuer avant, pendant et après la lecture et des informations par rapport aux aspects travaillés. Un signet suggérant des pistes d'exploitation additionnelles était aussi souvent ajouté. Certains ouvrages comprenaient également des rabats à soulever (intégrés dans le livre par l'équipe de recherche) révélant une question que le parent était invité à poser à son enfant à ce moment précis de la lecture. Enfin, des objets et des accessoires bonifiaient chacune des trousses afin que l'enfant et son parent puissent vivre des activités ludiques en lien avec la lecture du livre.

Les albums de littérature jeunesse ont été retenus en fonction de leur potentiel au regard du développement des compétences langagières et sociales par le biais de la lecture interactive. Les activités ludiques complémentaires ont aussi été élaborées en ce sens. Au plan langagier, les trousses visaient notamment à élargir le champ lexical, à travailler le schéma du récit, à développer l'habileté à réaliser des inférences et à développer la conscience phonologique et la correspondance graphème-phonème. Par rapport au développement social, les trousses abordaient les émotions, la résolution de conflits, le développement et le maintien des amitiés avec les pairs et soutenaient le développement de la capacité à prendre la perspective d'autrui (se mettre à la place de l'autre).

Chaque semaine, les enseignantes remettaient une nouvelle trousse aux enfants des GE. Ils en bénéficiaient pour une période de cinq jours, incluant la fin de semaine, afin de permettre aux parents de mieux l'intégrer à leur horaire. Afin de vérifier le bon état général des trousses avant que celles-ci ne soient redistribuées à de nouvelles familles, une assistante de recherche se rendait dans les milieux scolaires pour les réparer au besoin.

\section{Résultats}

Cette section rapporte les résultats associés aux réponses des parents en lien avec les deux objectifs poursuivis par la recherche.

\section{Résultats en lien avec l'implantation du programme auprès des parents}

Dans un premier temps, cette étude visait l'implantation du programme préconisé auprès des parents et l'accompagnement de ces derniers dans leurs interventions entourant l'exploitation de la littérature jeunesse. Le questionnaire soumis au post-test comprenait six 
questions permettant de recueillir des données susceptibles de fournir un éclairage sur l'atteinte de cet objectif.

L'une de ces questions invitait les parents à indiquer sur une échelle de type Likert à cinq niveaux, le nombre de trousses exploitées au cours du projet. La figure 1 présente le pourcentage de parents correspondant à chacun de ces cinq niveaux de réponses.

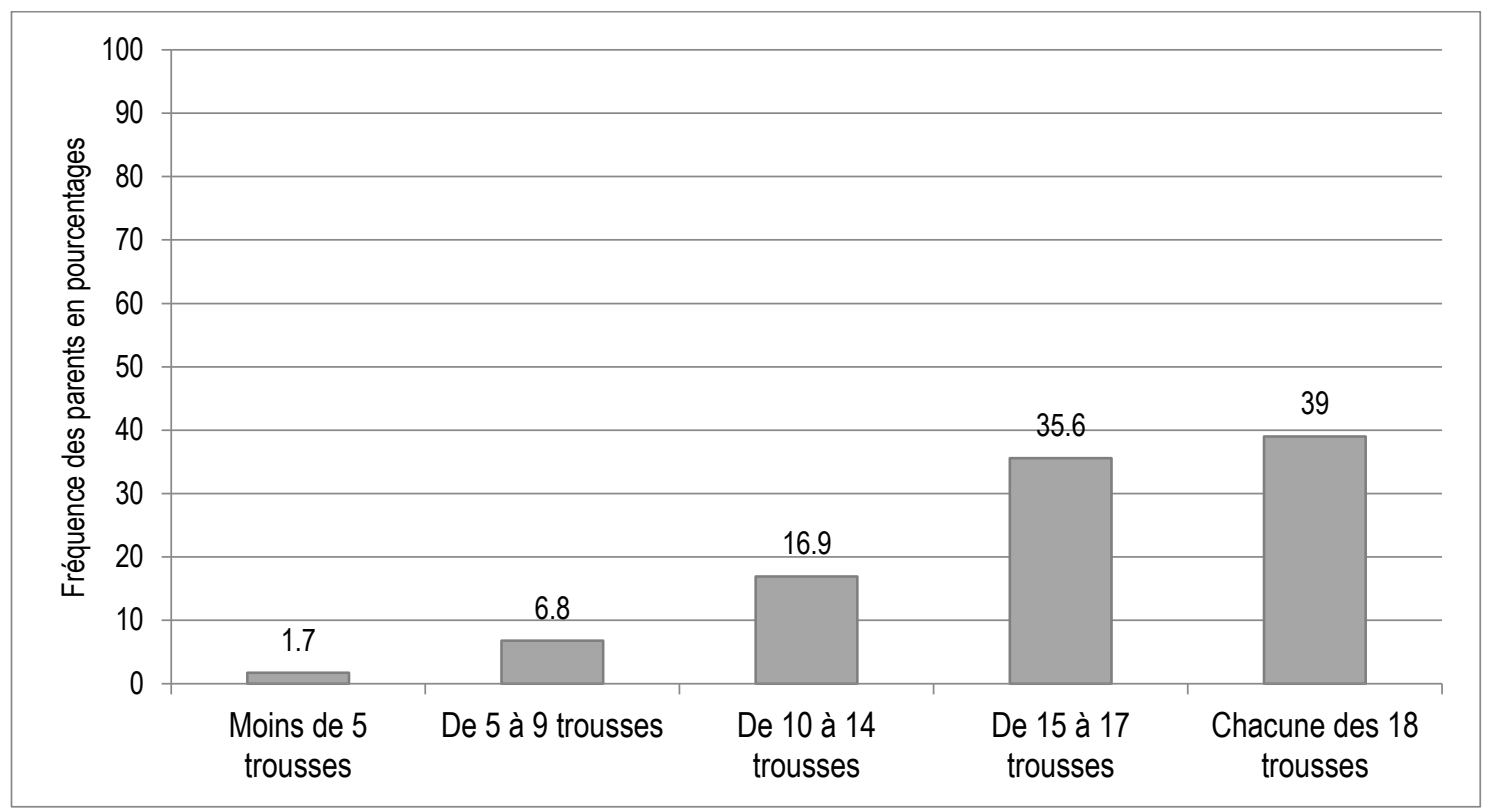

Figure 1. Nombre de trousses exploitées à la maison par les parents du GE $(\mathrm{n}=59)$

Les données recueillies montrent que $75 \%$ des parents ayant participé au projet affirment avoir exploité avec leur enfant la presque totalité des trousses proposées, soit entre 15 et 18 d'entre elles. Dix des 59 participants, soit $17 \%$ d'entre eux, déclarent avoir utilisé de 10 à 14 trousses. Enfin, seulement 8,5\% de l'ensemble des parents questionnés mentionnent avoir exploité moins de 10 des 18 trousses leur ayant été suggérées; un seul parmi eux énonce en avoir manipulé moins de cinq.

D'autre part, une autre question avait pour objectif d'amener les parents à estimer, sur une échelle de type Likert à quatre niveaux, le temps moyen investi chaque semaine dans l'exploitation des trousses. La figure 2 illustre le pourcentage de parents associé à chacun des quatre niveaux de temps d'exploitation des trousses. 


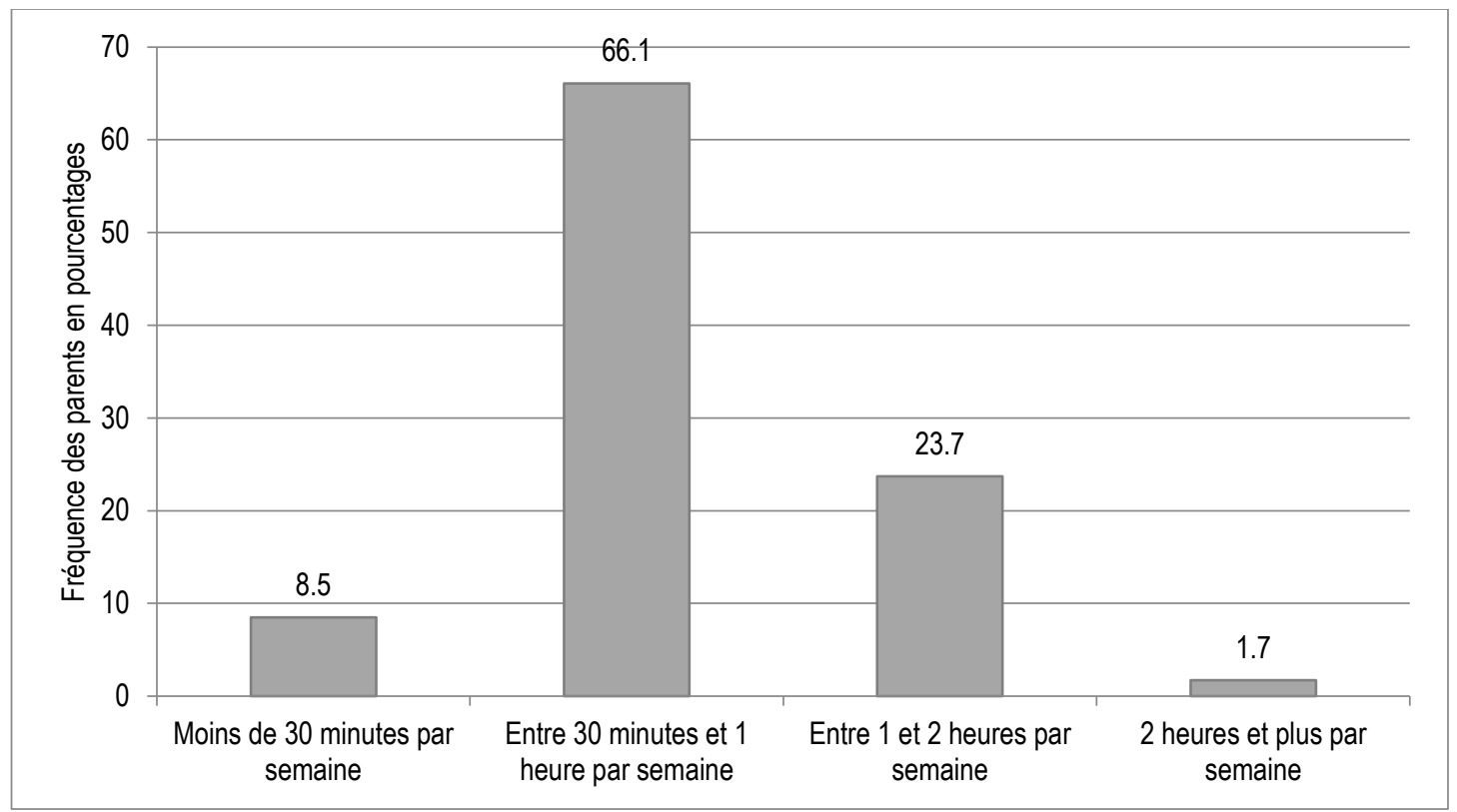

Figure 2. Estimation du temps consacré par semaine à l'exploitation des trousses par les parents du GE (n = 59)

Les données illustrées dans la figure 2 montrent que la majorité des parents, soit $66,1 \%$ d'entre eux, révèlent avoir consacré en moyenne entre 30 minutes et 1 heure par semaine à l'exploitation des trousses proposées. Il appert également que le quart des participants estime avoir passé en moyenne plus d'une heure par semaine à exploiter cellesci, alors que seulement 8,5\% disent y avoir consacré en moyenne moins de 30 minutes par semaine.

Le questionnaire demandait également aux parents d'exprimer, sur une échelle à quatre niveaux, à quel point ils avaient apprécié participer au projet, jugeaient que les formations leur avaient été utiles, avaient trouvé difficile ou lourd d'exploiter les trousses et estimaient que le projet avait modifié leur façon de faire la lecture avec leur enfant. La figure 3 rapporte les fréquences, en pourcentages, reliées aux réponses fournies par les parents à ces quatre questions. 


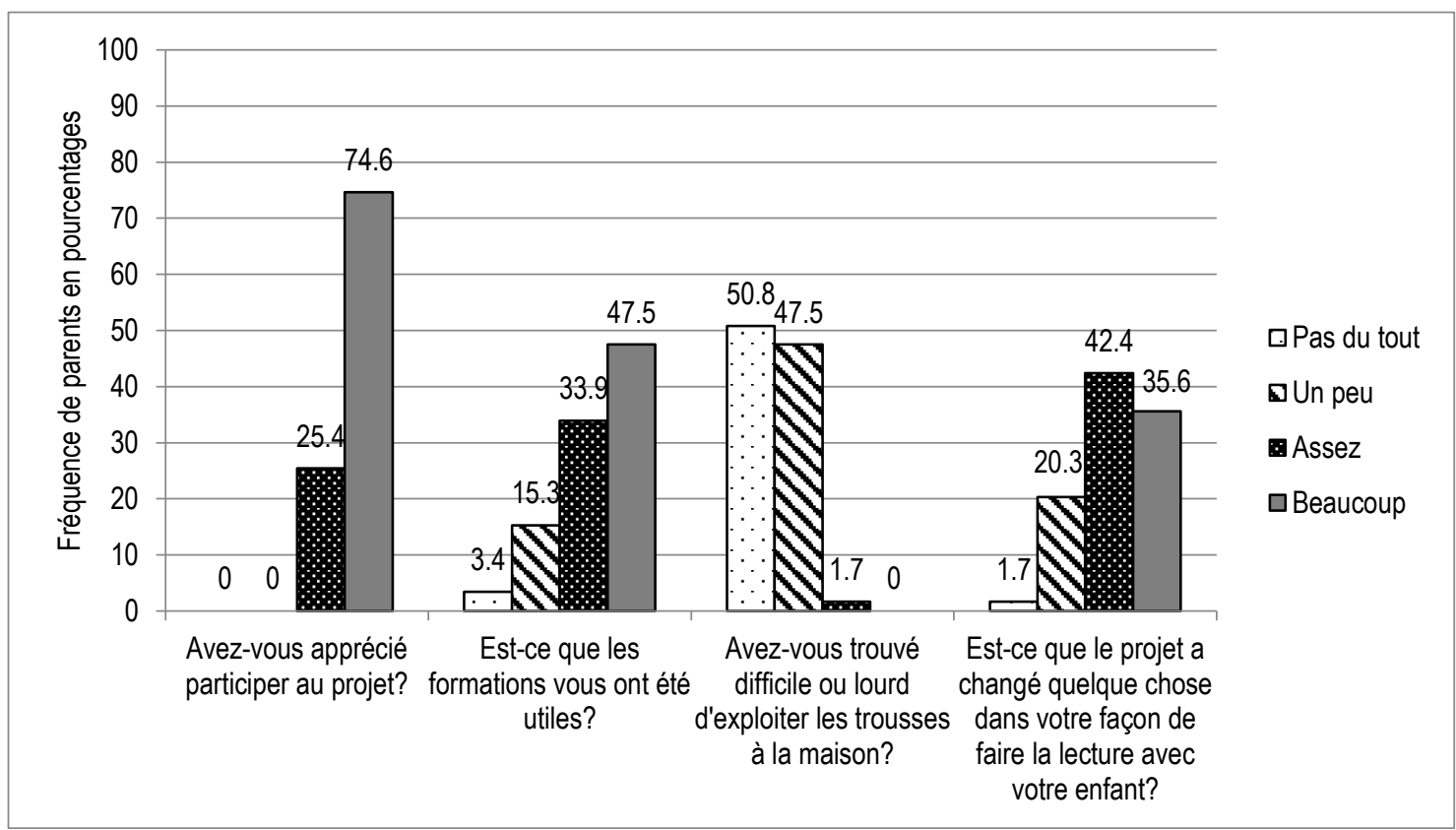

Figure 3. Fréquences, en pourcentages, relatives aux quatre questions en lien avec le niveau d'appréciation de la participation au projet par les parents du GE $(n=59)$

En ce qui concerne le niveau d'appréciation des parents à l'égard de leur participation au projet, les données sont éloquentes. La totalité des participants soutiennent avoir assez (25,4\%) ou beaucoup (74,6\%) apprécié leur expérience. Dans le même sens, les formations semblent avoir été jugées utiles par $96,7 \%$ des participants, soit un peu utiles par 15,3\% d'entre eux, assez utiles par 33\% d'entre eux et beaucoup utiles par 47,5\% d'entre eux. D'autre part, 98,3\% des parents révèlent n'avoir qu'un peu $(47,5 \%)$ ou pas du tout $(50,8 \%)$ trouvé l'exploitation des trousses difficile ou lourde. Enfin, le programme semble avoir contribué à modifier les pratiques de lecture de 98,3\% des participants, $20,3 \%$ de ceux-ci considérant que leurs pratiques ont changé un peu, 42,4\% assez et 35,6\% beaucoup.

Résultats concernant l'effet du programme sur la qualité des interventions parentales lors de la lecture interactive d'albums à leur enfant

Dans un deuxième temps, cette étude tentait de vérifier l'effet du programme proposé sur la qualité des interventions effectuées par les parents pour soutenir le développement du langage oral et écrit de leur enfant lors de la lecture interactive d'albums de littérature jeunesse. La figure 4 illustre la progression des scores moyens obtenus par les parents au prétest et au post-test aux 16 items du questionnaire évaluant la fréquence de leur recours à des interventions de qualité lorsqu'ils font la lecture interactive d'albums. 


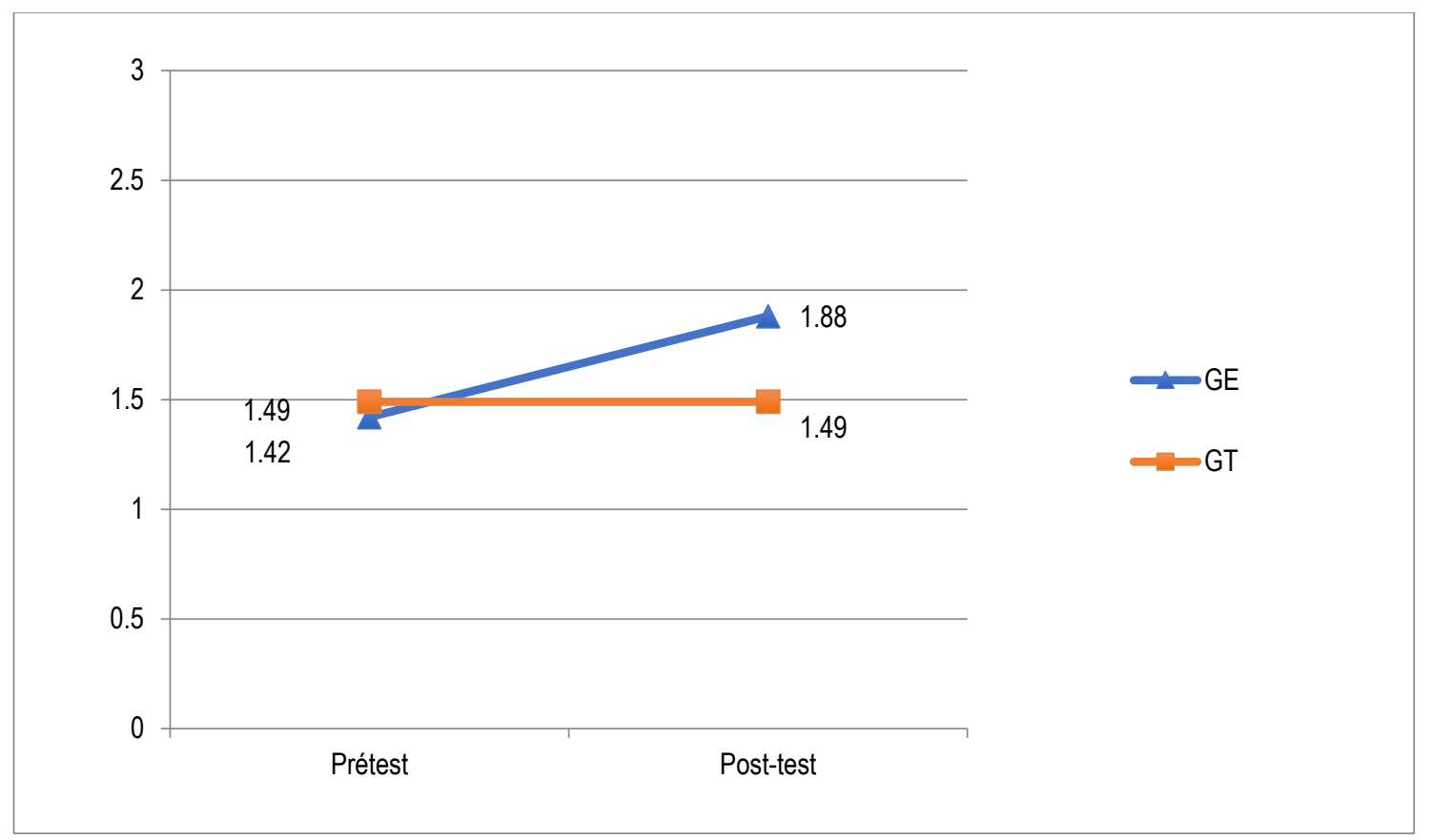

Figure 4. Moyennes des scores obtenus au prétest et au post-test par les parents du GE $(\mathrm{n}=59)$ et du GT $(\mathrm{n}=56)$ aux 16 items du questionnaire évaluant la fréquence de leur recours aux interventions de qualité lors de la lecture interactive d'albums (scores sur 3)

Les résultats de l'analyse de covariance effectuée entre les moyennes ajustées des scores au post-test du GE (M ajustée $=1,90$, Erreur-type $=0,050)$ et du GT (M ajustée $=1,47$, Erreur-type $=0,052$ ) révèlent la présence d'une différence statistiquement significative entre les deux groupes lorsque l'on tient compte des scores obtenus au prétest comme covariable $[\mathrm{F}(1,112)=34,870, \mathrm{p}<.001]$. La moyenne du GE est significativement supérieure à celle du GT, ce qui suggère que le programme aurait eu un effet positif sur la qualité des interventions des parents, conclusion que tendent à confirmer les résultats de l'analyse de variance à mesures répétées indiquant une différence significative entre les deux groupes sur le plan de la progression des scores moyens obtenus au prétest et au posttest $[\mathrm{F}(1,113)=30,404, \mathrm{p}<.001]$.

Parmi les 16 items du questionnaire précédemment soumis aux parents, 12 référaient à des interventions de type discussion autour du livre, deux correspondaient à des interventions explicitement orientées vers les concepts reliés à l'écrit et deux consistaient en des interventions sur le plan de la conscience phonologique. Des scores ont été calculés à partir des réponses fournies par les parents à ces trois catégories d'interventions. Les moyennes des scores obtenus au prétest et au post-test par les parents des deux groupes à ces trois catégories d'items sont présentées dans la figure 5. 


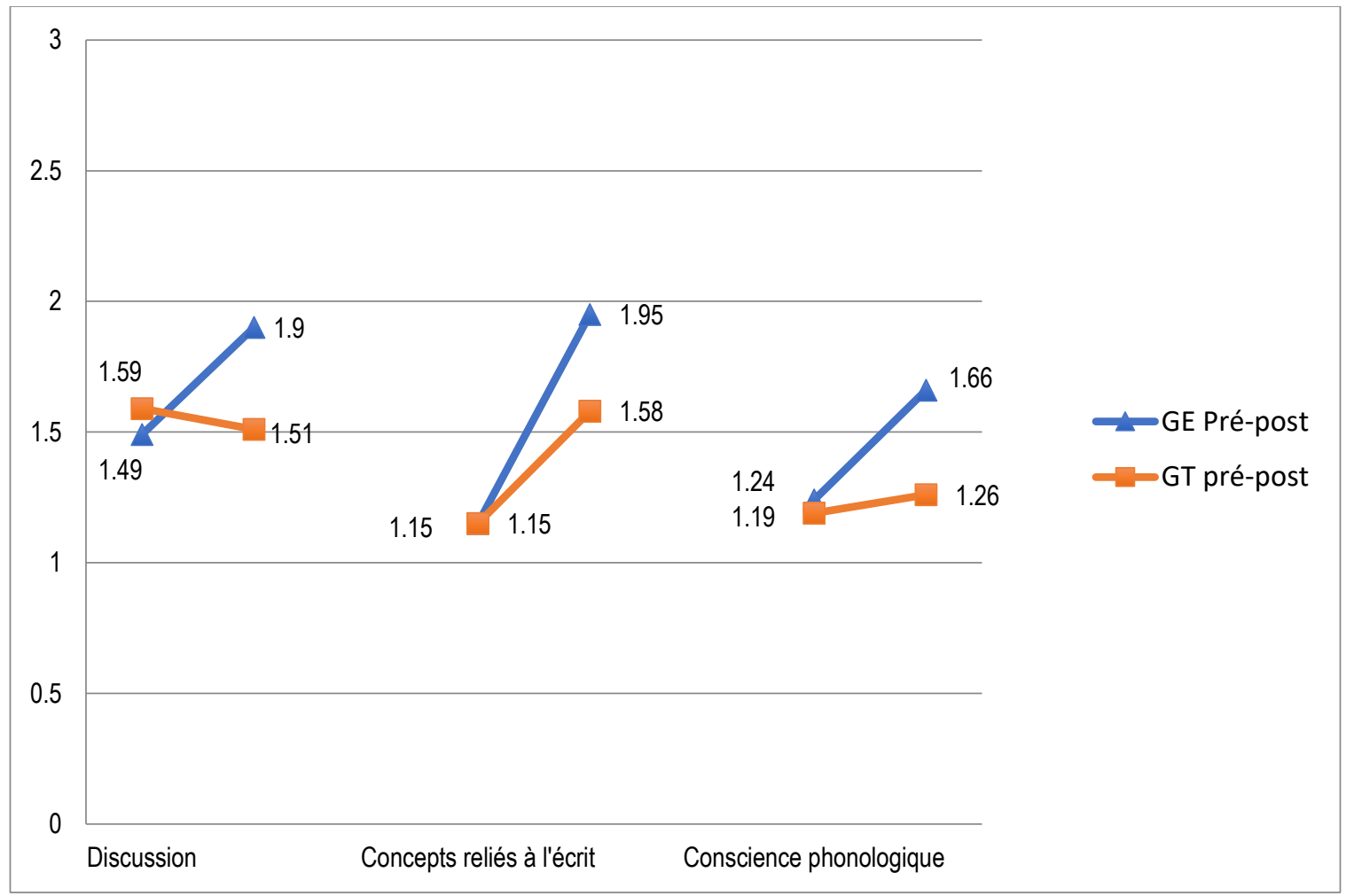

Figure 5. Moyennes des scores d'interventions de type discussion, d'interventions sur les concepts reliés à l'écrit et d'interventions axées sur la conscience phonologique obtenus au prétest et au post-test par le GE $(n=59)$ et le GT $(n=56)$ (scores sur 3$)$

Les résultats des analyses de covariance effectuées entre les moyennes ajustées des scores d'interventions de type discussion, d'interventions sur les concepts reliés à l'écrit et d'interventions axées sur la conscience phonologique obtenus au post-test par le GE et le GT en utilisant les scores du prétest comme covariable sont rapportés au tableau 1. Les résultats des analyses de covariance réalisées auprès de ces trois variables montrent que, pour chacune d'elles, les scores obtenus au post-test par les parents du GE s'avèrent, de façon statistiquement significative, supérieurs à ceux du GT lorsque l'on tient compte des scores obtenus au prétest (Interventions de type discussion : $[\mathrm{F}(1,112)=35,893, \mathrm{p}<.001]$; Interventions sur les concepts reliés à l'écrit : $[\mathrm{F}(1,112)=7,017, \mathrm{p}=.009]$; Interventions axées sur la conscience phonologique : $[\mathrm{F}(1,112)=8,942, \mathrm{p}=.003])$. De plus, les résultats des analyses de variance à mesures répétées indiquent la présence de différences statistiquement significatives entre le GE et le GT sur le plan de la progression des scores moyens obtenus au prétest et au post-test et cela, tant pour les interventions de type discussion autour du livre $[\mathrm{F}(1,113)=32,814, \mathrm{p}<.001]$, que pour celles portant sur les concepts reliés à l'écrit $[\mathrm{F}(1,113)=4,592, \mathrm{p}=.034]$ et sur la conscience phonologique $[\mathrm{F}(1,113)=5,830, \mathrm{p}=.017]$. De tels résultats révèlent que l'augmentation des scores du GE entre le prétest et le post-test s'avère statistiquement supérieure à celle des scores du GT, ce qui tend à montrer l'effet positif du programme sur les différents types d'interventions réalisées par les parents lors de la lecture interactive d'albums. 
Tableau 1

Résultats des analyses de covariance entre les moyennes ajustées des scores d'interventions de type discussion, d'interventions sur les concepts reliés à l'écrit et d'interventions sur la conscience phonologique obtenus au post-test par le $G E(n=59)$ et le $G T(n=56)$ en utilisant les scores du prétest comme covariable

\begin{tabular}{|c|c|c|c|c|c|c|c|}
\hline Variable & Groupe & $\begin{array}{c}\text { Moyenne } \\
\text { ajustée au } \\
\text { post-test } \\
\text { (score /3) }\end{array}$ & $\begin{array}{c}\text { Erreur } \\
\text {-type }\end{array}$ & MIN & MAX & $F$ & $p$ \\
\hline $\begin{array}{l}\text { Interventions de type } \\
\text { discussion }\end{array}$ & $\begin{array}{l}\text { Expérimental } \\
\text { Témoin }\end{array}$ & $\begin{array}{l}1,93 \\
1,48\end{array}$ & $\begin{array}{l}0,052 \\
0,053\end{array}$ & $\begin{array}{l}0,92 \\
0,17\end{array}$ & $\begin{array}{l}3,00 \\
2,75\end{array}$ & $35,893^{1}$ &, $000 * * *$ \\
\hline $\begin{array}{l}\text { Interventions sur les } \\
\text { concepts reliés à l'écrit }\end{array}$ & $\begin{array}{l}\text { Expérimental } \\
\text { Témoin }\end{array}$ & $\begin{array}{l}1,95 \\
1,58\end{array}$ & $\begin{array}{l}0,097 \\
0,100\end{array}$ & $\begin{array}{l}0,00 \\
0,00\end{array}$ & $\begin{array}{l}3,00 \\
3,00\end{array}$ & 7,017 &, $009 * *$ \\
\hline $\begin{array}{l}\text { Interventions sur la } \\
\text { conscience } \\
\text { phonologique }\end{array}$ & $\begin{array}{l}\text { Expérimental } \\
\text { Témoin }\end{array}$ & $\begin{array}{l}1,65 \\
1,27\end{array}$ & $\begin{array}{l}0,088 \\
0,090\end{array}$ & $\begin{array}{l}0,00 \\
0,00\end{array}$ & $\begin{array}{l}3,00 \\
3,00\end{array}$ & 8,942 &, $003 * *$ \\
\hline
\end{tabular}

Note ${ }^{1}$ Il est à noter que les résultats de l'ANCOVA effectuée à partir des scores relatifs aux interventions de type discussion doivent être considérés avec prudence, car le postulat d'homogénéité des pentes n'est pas respecté $(\mathrm{p}=.033)$. Par conséquent, pour assurer la validité des résultats, deux tests-t ont été calculés. Les résultats du premier test-t effectué à partir des scores du prétest indiquent que les moyennes des deux groupes ne présentent pas de différences statistiquement significatives entre elles $[\mathrm{t}(1,113)=0,973, \mathrm{p}=.332]$. Pour cette variable, les scores du prétest ont donc peu d'impact sur les différences remarquées au post-test à l'aide du deuxième test-t, qui confirme que la moyenne des scores du groupe expérimental est significativement plus élevée que celle du groupe témoin $[\mathrm{t}(1,113)=4,03, \mathrm{p}<.001]$. Les résultats des test-t vont dans le même sens que ceux de l'ANCOVA.

$* * \mathrm{p}<.01 ; * * * \mathrm{p}<.001$.

\section{Discussion}

Cette étude avait comme premier objectif d'implanter le programme Les mille et un plaisirs de lire avec toi et d'accompagner les parents du GE dans leurs interventions entourant l'exploitation de la littérature jeunesse. Les résultats obtenus concernant le nombre de trousses littéraires que les parents ont exploité à la maison tout au long de l'expérimentation et le temps moyen investi chaque semaine dans leur exploitation révèlent qu'ils se sont fortement engagés dans le projet. En effet, $75 \%$ des parents affirment avoir exploité entre 15 et la totalité des 18 trousses, et 66,1\% estiment avoir consacré en moyenne entre 30 minutes et 1 heure par semaine à leur exploitation alors que 25,4\% des parents révèlent y avoir passé en moyenne plus d'une heure par semaine. Ces résultats soutiennent l'idée que l'implantation du projet au sein des familles s'est effectuée conformément à nos attentes. On peut penser que ce dernier a plu aux parents, possiblement parce qu'il répondait à leurs besoins et intérêts (Deslandes \& Bertrand, 2001). De plus, le fait qu'il ait tenu compte des recommandations de Beauregard et de ses collaboratrices (2011) (présentation des objectifs du projet, parole donnée régulièrement aux parents, approche basée sur le plaisir, ajustement des ateliers à l'horaire des familles, etc.) a pu contribuer à favoriser l'engagement des participants et leur persévérance dans le projet. 
Quant à leur appréciation concernant leur participation au programme, les résultats sont éloquents. La totalité des participants soutiennent avoir assez ou beaucoup apprécié leur expérience. Les trois ateliers de formation auxquels ils ont assisté ont été jugés utiles par 96,7\% d'entre eux. Cette formule d'accompagnement sous forme d'ateliers, incluant une modélisation de pratiques, semble donc plaire aux parents et apparait répondre à leurs besoins. Ces observations vont dans le même sens que celles de Giasson et Saint-Laurent (2004), Charron et ses collaboratrices (2013) et Boudreau (2017) qui recommandaient cette formule pour former les parents aux pratiques à adopter pour soutenir, notamment, les compétences langagières de leur enfant.

De plus, la formule des trousses littéraires proposée par le programme n'a pas été perçue comme difficile ou lourde à exploiter par les parents qui ont participé au projet. Dans un contexte où la lecture d'histoires ne semble pas une pratique fréquente dans les familles (Lavoie \& Fontaine, 2016), ces données soutiennent la pertinence d'offrir aux parents du matériel pour soutenir et enrichir leurs interventions quotidiennes afin qu'ils deviennent plus assidus et mieux outillés dans leur démarche éducative (Britto \& BrooksGunn, 2001; Thériault \& Lavoie, 2004).

L'étude poursuivait également un second objectif, soit celui d'évaluer l'effet du programme Les mille et un plaisirs de lire avec toi sur la qualité des interventions des parents lors de la lecture interactive d'albums pour favoriser le développement des compétences langagières de leur enfant. À cet égard, 98,3\% des parents rapportent que le programme a modifié leur façon de faire la lecture avec leur enfant. De surcroit, les résultats obtenus révèlent qu'à la suite de leur participation au programme, les parents du GE affirment recourir plus souvent aux différentes interventions de qualité proposées dans le questionnaire pour soutenir les compétences langagières de leur enfant lors de la lecture interactive d'albums que ceux du GT. En effet, ils soutiennent réaliser plus fréquemment des interventions permettant de discuter autour du livre, d'interventions orientées vers les concepts de lettres et de mots et d'interventions favorisant la conscience phonologique que ne disent le faire les parents du GT. Sur ce plan, les analyses révèlent également que les changements de pratiques observés entre le prétest et le post-test sont significativement différents entre les deux groupes de parents, et cela, en faveur des parents du GE chez qui l'augmentation du nombre d'interventions de qualité déclarées entre le début et la fin du projet se révèle statistiquement supérieure à celle observée auprès des parents du GT chez qui les scores ont moins ou, dans certains cas, pas du tout progressé. On peut penser qu'il s'agit là d'un effet du programme mis en place, les ateliers de formation s'étant particulièrement attardés aux interventions à privilégier avant, pendant et après la lecture de l'album, ainsi qu'à l'importance de profiter des occasions pour discuter avec l'enfant des lettres et des sons par exemple.

Plus spécifiquement, en ce qui concerne les interventions de type discussion autour du livre, il s'avère particulièrement intéressant de noter la différence statistiquement significative entre l'augmentation des pratiques déclarées des parents du GE et la baisse de pratiques déclarées par les parents du GT. Ces interactions parent-enfant durant la lecture s'avèrent précieuses, car elles sont au cœur du développement des compétences langagières de l'enfant (Dionne, 2013; Mol et al., 2009). Les résultats montrent, notamment, que les parents du GE du présent échantillon déclarent, au terme du projet, questionner davantage leur enfant que ceux du GT. De telles pratiques de dialogue autour du livre sont recommandées pour favoriser l'engagement de l'enfant et ainsi mieux soutenir sa 
compréhension du texte (Dougherty Stahl, 2014), le développement de son vocabulaire (Wasik et al., 2016), la compréhension de nouveaux concepts (Zucker et al., 2010) et l'émergence de l'écrit (Justice \& Ezell, 2002; Swanson et al., 2011). On peut penser que le programme Les mille et un plaisirs de lire avec toi, de par les nombreux moyens utilisés pour les soutenir dans leurs lectures interactives (modelage, signets avec questions à poser à l'enfant et rabats ajoutés dans certains livres pour susciter les échanges, etc.), aura permis aux parents de prendre conscience de l'importance de la discussion autour du livre pour favoriser le développement langagier de leur enfant.

En somme, les résultats obtenus dans le cadre de cette recherche appuient l'importance de former les parents, ce que recommandent plusieurs auteurs (Baker, 2013; Boudreau, 2017; Myre-Bisailllon et al., 2014; Saint-Laurent \& Giasson, 2005). En effet, les interventions des parents sont souvent intuitives (Baker, 2013; Ziv et al., 2014) et le modelage vise à les guider de façon plus explicite. On peut penser que les trois ateliers de formation proposés dans cette recherche ainsi que les 18 trousses littéraires auxquelles ils ont eu accès pendant six mois ont bien accompagné les parents en les exposant à des connaissances et des stratégies qu'ils déclarent s'être appropriées.

Cette recherche quasi expérimentale se distingue d'autres travaux portant sur des programmes de littératie familiale, car elle adopte un devis impliquant un groupe expérimental et un groupe témoin, ce qui est peu fréquent dans les programmes de littératie familiale recensés (Sénéchal, 2008). Ceci constitue une force de l'étude. Or, la recherche présente aussi des limites qu'il convient de considérer. En effet, les données présentées dans cet article sont issues d'un questionnaire autodéclaré, ce qui peut représenter une limite de la recherche. D'une part, le format des questions fermées ne permettait pas aux parents d'illustrer les pratiques qu'ils adoptent autant que ne l'auraient permis des questions ouvertes (ex. : pouvoir préciser le type de questions qu'ils posent à leur enfant lors de leurs interventions autour du livre). Les pratiques déclarées par les parents gagneraient aussi à être confrontées à l'observation des pratiques effectives vécues à la maison. L'ajout d'instruments de mesure complémentaires (ex. : filmer les parents en situation de lecture interactive d'albums) permettrait d'éviter les biais associés à la désirabilité sociale.

\section{Conclusion}

L'intérêt de chercher des moyens d'aider et de soutenir les parents afin qu'ils deviennent plus compétents pour aider leur enfant ne fait pas de doute (Tamis-LeMonda \& Rodriguez, 2014). Myre-Bisaillon et ses collaborateurs (2010) rappellent que, parmi les interventions préventives en petite enfance, peu de programmes sont directement destinés aux parents. Les constats qui émergent de notre recherche appuient l'idée que les ateliers de formation et les trousses littéraires, qui visaient justement à outiller et accompagner les parents pour favoriser le développement des compétences langagières de leur enfant à l'aide de la lecture interactive d'albums de littérature jeunesse, sont appréciés des parents et peuvent conduire à un enrichissement de leurs pratiques de littératie familiale. Devant l'importance que revêt la lecture interactive d'albums pour soutenir le développement du langage oral et écrit du jeune enfant (Dionne, 2013; Kispal, 2008, Makdissi et al., 2010; Montesinos-Gelet et al., 2015; Saracho, 2017), accompagner les parents à saisir le rôle déterminant qu'ils peuvent jouer auprès de leur enfant à cette période sensible pourrait 
éviter que des problèmes scolaires ne prennent des proportions importantes, et ce, particulièrement en milieux défavorisés (Boudreau \& Grondin, 2012; Irwin et al., 2007).

Par ailleurs, en sachant que les parents qui ont participé à la présente étude déclarent avoir modifié leur façon d'accompagner leur enfant lors de la lecture interactive d'albums, un suivi longitudinal serait pertinent pour vérifier si ces pratiques se maintiennent dans le temps. En cherchant à décrire et comprendre les effets à plus long terme du présent programme, il serait possible de mieux documenter l'apport des ateliers de formation et des trousses littéraires à entrainer de réelles transformations des pratiques de littératie familiale.

Enfin, le programme Les mille et un plaisirs de lire avec toi présenté ici pourrait inspirer les différents acteurs des milieux scolaires (conseillers pédagogiques, enseignants de maternelle 4 et 5 ans), des centres de la petite enfance et des fondations pour l'alphabétisation afin d'alimenter leur réflexion sur la façon dont ils souhaitent accompagner et soutenir les parents, particulièrement ceux qui ont des enfants d'âge préscolaire. Considérant le rôle central que jouent les parents dans la réussite éducative de leur enfant (Sénéchal, 2008; Beauregard et al., 2011) et l'importance de bien les former pour enrichir leurs interventions (Myre-Bisaillon et al., 2010; Ziv et al., 2014), on ne peut que recommander aux instances politiques de déployer les ressources nécessaires afin de rendre des programmes de littératie familiale accessibles au plus grand nombre et de voir à en assurer la pérennité.

\section{Références}

Aràm, D., Fine, Y., \& Ziv, M. (2013). Enhancing parent-child shared book reading interactions: Promoting references to the book's plot and socio-cognitive themes. Early Childhood Research Quarterly, 28, 111-122.

Baker, C. E. (2013). Fathers' and mothers' home literacy involvement and children's cognitive and social emotional development: implications for family literacy programs. Applied developmental science, 17(4), 184-197.

Beauregard, F., Carrignan, I., \& Létourneau, M.-D. (2011). Recension des écrits scientifiques sur la littératie familiale et communautaire. Ministère de l'Éducation, du Loisir et du Sport. Repéré à :

https://depot.erudit.org/bitstream/003789dd/1/Beauregard_Carignan_MELS_litterati e_familiale.pdf

Blachowicz, C. L. Z., \& Fisher, P. J. (2015). Best Practices in Vocabulary Instruction, in: L. B. Gambrell \& L. M. Morrow (Eds.), Best Practices in Literacy Instruction (5th Edition) (pp. 195-222). New York : The Guilford Press.

Boisvert, G., \& Gagnon, J. (2005). Éveiller l'enfant à l'écrit : de la naissance à l'école. Montréal : Hurtubise HMH.

Boivin, M., Hertzman, C., Barr, R.G., Boyce W.T., Fleming, A., MacMillan, H., Odgers, C., Sokolowski, M.B., \& Trocmé, N. (2012). Early childhood development. Ottawa: The Royal Society of Canada \& The Canadian Academy of Health Sciences Expert Panel.

Boudreau, M. (2005). La littératie familiale et le développement de la conscience phonologique chez les enfants de maternelle. Thèse de doctorat inédite, Université Laval. 
Boudreau, M. (2017). La littératie familiale : pour amorcer l'aventure de la lecture et de l'écriture du bon pied. Revue préscolaire, 55(1), 10-12.

Boudreau, M., \& Grondin, J. (2012). Programme de stimulation parentale à la conscience phonologique et aux lettres de l'alphabet auprès de parents d'enfants de maternelle : résultats de la version préliminaire. Revue pour la recherche en éducation, 2, 50-67.

Boudreau, M., Saint-Laurent, L., \& Giasson, J. (2009). La littératie familiale et ses liens avec la conscience phonologique, l'émergence de l'écrit et le vocabulaire des enfants de maternelle, in: D. Masny (Eds.), Lire le monde: les littératies multiples et l'éducation dans les communautés francophones (pp.187-238). Ottawa: Les Presses de l'Université d'Ottawa.

Britto, P. R., \& Brooks-Gunn, J. (2001). The role of family literacy environments in promoting young children's emerging literacy skills. San Francisco : Jossey-Bass.

Brodeur, M., Gosselin, C., Mercier, J. Legault, F., \& Vanier, V. (2006). Prévention des difficultés d'apprentissage en lecture : l'effet différencié d'un programme implanté par des enseignantes de maternelle chez leurs élèves. Éducation et francophonie, 34(2), $56-83$

Cantin, G., Bouchard, C., \& Charron, A. (2010). Conclusion. Pour soutenir le développement du langage et de la littératie. Quelques balises à retenir, in : A. Charron, C. Bouchard \& G. Cantin (Eds.) Langage et littératie chez l'enfant en service de garde éducatif (pp. 175-186). Québec : Presses de l'université du Québec.

Charron, A., Gagnon, B., \& Fortin-Clément, G. (2013). Bilan du projet de rechercheaction: Il était une fois... un livre pour toi et moi. Récupéré à http://rire.ctreq.qc.ca/wp-content/uploads/2017/06/quipe-de-recherche_StVincent_Rapport-Il-e\%CC\%81tait-une-fois.pdf

Conseil supérieur de l'éducation (CSÉ) (2012). Mieux accueillir et éduquer les enfants d'âge préscolaire, une triple question d'accès, de qualité et de continuité des services. Québec : Gouvernement du Québec.

Côté, G. (2011). Sans toi!. Markham : Éditions Scholastic.

Deslandes, R., \& Bertrand, R. (2001). La création d'une véritable communauté éducative autour de l'élève : une intervention plus cohérente et des services mieux harmonisés. Rapport de recension des écrits, CQRS-MEQ action concertée. Repéré à http://crires.ulaval.ca/financement/la-creation-dune-veritable-communauteeducative-autour-de-leleve-une-intervention-plus\#

Desrosiers, H., \& Ducharme, A. (2006). Commencer l'école du bon pied. Facteurs associés à l'acquisition du vocabulaire à la fin de la maternelle. Étude longitudinale du développement des enfants du Québec (ÉLDEQ 1998-2010). Institut de la statistique du Québec, 4(1), 1-16.

Dionne, A.M. (2013). Le plaisir de lire avec son enfant : un enjeu déterminant pour une pratique de littératie familiale aux multiples facettes. Forumlecture.ch, (3). Repéré à http://www.forumlecture.ch/myUploadData/files/2013_3_Dionne.pdf

Domitrovich, C. E., Bradshaw, C.P., Greenberg, M. T., Embry, D., Poduska, J. M., \& Ialongo, N. S. (2010). Integrated models of school-based prevention : Logic and theory. Psychology in School, 47(1), 71-88.

Dougherty Stahl, K. A. (2014). Fostering Inference Generation With Emergent and Novice Readers. Reading Teacher, 67(5), 384-388. 
Dupin de Saint-André, M. (2011). L'évolution des pratiques de lecture à haute voix d'enseignantes expertes et leur influence sur le développement de l'habileté des élèves du préscolaire à faire des inférences. Thèse de doctorat, Université de Montréal.

Dupin de Saint-André, M., Montésinos-Gelet, I., \& Morin, M.-F. (2012). Influence des pratiques de lecture à haute voix d'enseignantes expertes sur le développement de l'habileté à faire des inférences d'élèves du préscolaire. Lettrure 2, 22-49.

Duncan, G. J., \& Magnuson, K. (2013). Investing in Preschool Programs. The Journal of Economic Perspectives : a journal of the American Economic Association, 27(2), 109132.

Duncan, G. J., \& Sojourner, A. J. (2013). Can Intensive Early Childhood Intervention Programs Eliminate Income-Based Cognitive and Achievement Gaps?, Journal of Human Resources, 48(4), 945-968.

Ehri, L.C., Nunes, S.R., Stahl, S.A., \& Willows, D.M. (2001). Systematic phonics instruction helps students learn to read : Evidence from the National Reading Panel's meta-analysis. Review of Education, 71(3), 393-447.

Fayol, M. (2017). Les premiers apprentissages. Mieux les décrire pour mieux prévenir et mieux intervenir. Administration et Éducation, 3(155), 103-108.

Foulin, J.N., \& Pacton, S. (2006). La connaissance du nom des lettres : précurseur de l'apprentissage du son des lettres. Éducation et francophonie, 34(2), 28-55.

Giasson, J. (1995). La lecture : de la théorie à la pratique. Boucherville : Gaëtan Morin Éditeur.

Giasson, J., \& Saint-Laurent, L. (2004). Facteurs de protection contre l'échec en lecture au premier cycle du primaire. Rapport synthèse, Université Laval.

Godin, M.-P., Godard, L., Chapleau, N., \& Gagné, A. (2015). La lecture interactive d'albums pour les élèves du préscolaire ayant des difficultés langagières: une intervention prometteuse pour améliorer le vocabulaire. Language and Literacy, 17(3), 34-59

Hart, B., \& Risley, T.R. (2003). The Early Catastrophe : The 30 Million Word Gap by Age 3. American educator, 27(1), 4-9.

Irwin, L., Siddiqi, A., \& Hertzman, C. (2007). Le développement de la petite enfance : un puissant égalisateur. Rapport final. Commission des déterminants sociaux de la santé de l'Organisation mondiale de la Santé.

Janosz, M., Pascal, S., Belleau, L., Archambault, I., Parent, S., \& Pagani, L. (2013). Les élèves du primaire à risque de décrocher au secondaire : caractéristiques à 12 ans et prédicteurs à 7 ans. Étude longitudinale du développement des enfants du Québec (ÉLDEQ 1998-2010). Institut de la statistique du Québec, 7(2).

Justice, L.M., \& Ezell, H. K. (2002). Use of storybook reading to increase print awareness in at-risk children. American Journal of Speech-language Pathology, 11(1), 17-29.

Jung, E. (2016). The Development of Reading Skills in Kindergarten Influence of Parental Beliefs About School Readiness, Family Activities, and Children's Attitudes to School. Revue Internationale de l'Enfant Préscolaire. 48(1), 61-78.

Kispal, A. (2008). Effective Teaching of Inference Skills for Reading : Literature Review : (Research Report DCSF-RR031). National Foundation for Educational Research.

Lavoie, A., \& Fontaine, C. (2016). Mieux connaître la parentalité au Québec : Un portrait à partir de l'Enquête québécoise sur l'expérience des parents d'enfants de 0 à 5 ans 2015. Québec : Institut de la statistique du Québec. 
Makdissi, H., Boisclair, A., \& Sirois, P. (2010). La littératie au préscolaire; une fenêtre ouverte vers la scolarisation. Québec : Presses de l'Université du Québec.

Maltais, C. (2007). Relation entre la littératie familiale en milieu francophone et l'incidence d'un programme de maternelle quatre ans à temps plein sur le développement $\mathrm{du}$ langage et de la lecture des enfants. Éducation francophone en milieu minoritaire, 2(1), 6-18.

Marmot, M.G., Allen, J., Goldblatt, P., Boyce, T., McNeish, D., Grady, M., \& Geddes, I. (2010). Fair society, healthy lives: Strategic review of health inequalities in England post-2010. The Marmot Review. London : Institute of Health Inequalities.

Marulis, L. M., \& Neuman, S. B. (2010). The effects of vocabulary intervention on young children's word learning : a meta-analysis. Review of Educational Research, 80(3), 300-335.

Masny, D. (2008). Main dans la main: la littératie familiale en milieu minoritaire. Ottawa: Coalition francophone pour l'alphabétisation et la formation de base en Ontario.

Ministère de l'Éducation du Québec (MEQ) (2003). Les difficultés d'apprentissage à l'école. Cadre de référence pour guider l'intervention. Québec : Gouvernement du Québec.

Ministère de l'Éducation et de l'Enseignement Supérieur (MEES) (2015). Indices de défavorisation 2015-2016. Repéré à

http://www.education.gouv.qc.ca/fileadmin/site_web/documents/PSG/statistiques_in fo_decisionnelle/Indices_PUBLICATION_20152016.pdf.

Mol, S. E., Bus, A. G., \& de Jong, M. T. (2009). Interactive book reading in early education : A tool to stimulate print knowledge as well as oral language. Review of Educational Research, 79, 979-1008.

Montésinos-Gelet, I., Dupin de Saint-André, M., \& Bourdeau, R. (2015). Accompagner des élèves en difficulté dans leur appropriation de l'écrit à l'aide de la littérature jeunesse. Revue de Recherches en LMM, 2, 1-50.

Morris, L., Godard, L., Labelle, L., Simard, D., Sabourin, C., \& Roy, C. (2008). Une juste mesure: Développement d'instruments et de critères d'évaluation linguistique pour des élèves allophones. Rapport de recherche.

Mourlevat, J.-C., \& Bénazet, J.-L. (1998). Le jeune loup qui n'avait pas de nom. Toulouse : Milan.

Myre-Bisaillon, J., Boutin, N., \& Beaudoin, C. (2014). Les pratiques d'éveil à la lecture et à l'écriture à la maternelle en milieux défavorisés : quand les parents viennent en classe. Nouveaux cahiers de la recherche en éducation, 17(2), 66-95.

Myre-Bisaillon, J., Villemagne, C., Puentes-Neuman, G., Raîche, G., Dionne, C., \& Louis, R. (2010). Évaluation des impacts du Programme d'aide à l'éveil à la lecture et à l'écriture dans les milieux défavorisés : Rapport de recherche final. Québec : Fonds québécois de recherche sur la société et la culture (FRQSC). Repéré à http://www.frqsc.gouv.qc.ca/fr/partenariat/nos-resultats-de recherche/histoire/evaluation-des-impacts-du-programme-d-eveil-a-la-lecture-et-a-1ecriture-paele-dans-les-milieux-defavorises-2mzv6h871429817638265

Pagani, L. S., Fitzpatrick, C., Belleau, L., \& Janosz, M. (2011). Prédire la réussite scolaire des enfants en quatrième année à partir de leurs habiletés cognitives, comportementales et motrices à la maternelle. Dans Étude longitudinale du 
développement des enfants du Québec (ÉLDEQ 1998-2010) - De la naissance à 10 ans. Québec : Institut de la statistique du Québec.

Poissant, J., \& Garnache, L. (2016). Analyse contextualisée sur le développement des enfants à la maternelle. Québec: Institut national de santé publique du Québec (INSPQ).

Puentes-Neuman, N., Myre-Bisaillon, J., Collette, K., Larose, F., \& Morin, P. (2015). Rapport de recherche, programme actions concertées; Quel soutien scolaire et communautaire offrir aux parents afin de favoriser la réussite de l'entrée dans l'écrit chez les enfants? (Projet de recherche $\mathrm{n}^{\circ}$ 2010-ER-136894). Sherbrooke : Université de Sherbrooke.

Saint-Laurent, L., \& Giasson, J. (2005). Effects of a Family Literacy Program Adapting Parental Intervention to First Graders' Evolution of Reading and Writing Abilities. Journal of Early Childhood Literacy, 5(3), 253-278.

Saracho, O. N. (2017). Parents' shared storybook reading - learning to read. Early child development and care, 187(3-4), 554-567.

Sénéchal, M., \& LeFevre, J. (2002). Parental involvement in the development of children's reading skill : A five-year longitudinal study. Child Development, 73(2), 445-460.

Sénéchal, M. (2008). L'effet d'interventions familiales sur l'acquisition de la lecture par les enfants: de la maternelle à la 3e année. Encyclopédie du développement du langage et de l'alphabétisation. (p. 1-8). London, Ontario : Réseau canadien de recherche sur le langage et l'alphabétisation. Repéré à

http://www.literacyencyclopedia.ca/pdfs/topic.php?topId=251\&fr=true

Simard, M., Tremblay, M.-Ė., Lavoie, A., \& Audet, N. (2013). Enquête québécoise sur le développement des enfants à la maternelle (EQDEM) 2012. Québec : Institut de la statistique du Québec.

Swanson, E.A., Wanzek, J., Petscher, Y., Vaughn, S., Heckert, J., Cavanaugh, C., Kraft, G., \& Tackett, K. (2011). A synthesis of read-aloud interventions on early reading outcomes among preschool through third graders at risk for reading difficulties. Journal Learning Disabilities, 44(3), 258-275.

Tamis-LeMonda, C., \& Rodriguez, E.T. (2014). Rôle des parents pour favoriser l'apprentissage et l'acquisition du langage chez les jeunes enfants. Encyclopédie sur le développement des jeunes enfants. Habiletés parentales ( $3^{\mathrm{e}}$ éd.). Repéré à http://www.enfant-encyclopedie.com/sites/default

Taylor, C.L., Zubrick, S.R., \& Christensen, D. (2016). Barriers to Parent-Child Book Reading in Early Childhood. Revue Internationale de l'Enfant Préscolaire, 48(3), 295309.

Thériault, J., \& Lavoie, N. (2004). L'éveil à la lecture et à l'écriture : une responsabilité familiale et communautaire. Montréal : Les Éditions Logiques.

Wasik, B. A., Hindman, A.H., \& Snell, E. K. (2016). Book reading and vocabulary development : A systematic review. Early Childhood Research Quarterly, 37(4), 3957.

Ziv, M., Smadja, M., \& Aràm, D. (2014). Mothers and teachers' mental-state discourse with preschoolers during storybook reading. Journal of cognitive education and psychology, 13(1), 103-119. 
Zucker, T.A., Justice, L.M., Piasta, S.B., \& Kaderavek, J.N. (2010). Preschool teachers' literal and inferential questions and children's responses during whole-class shared. Early childhood research quarterly, 25(1), 65-83.

Biographie des auteures

Monica Boudreau, Ph. D., Professeure-chercheuse en éducation préscolaire à l'Unité départementale des sciences de l'éducation de l'Université du Québec à Rimouski (UQARLévis). Ses intérêts de recherche portent sur l'émergence de l'écrit, la littératie familiale, la littérature jeunesse et les pratiques enseignantes. monica_boudreau@ uqar.ca

Isabelle Beaudoin, $\mathrm{Ph}$. D. Professeure-chercheuse en didactique du français/adaptation scolaire à l'Unité départementale des sciences de l'éducation de l'Université du Québec à Rimouski (UQAR-Lévis). Ses intérêts de recherche portent sur l'émergence de l'écrit et la didactique du français au primaire, la prévention des difficultés en lecture et en écriture et la littérature jeunesse. isabelle_beaudoin@uqar.ca

Julie Mélançon, Ph. D., Professeure-chercheuse en développement de l'enfant à l'Unité départementale des sciences de l'éducation de l'Université du Québec à Rimouski (UQARLévis). Ses intérêts de recherche portent sur le développement de l'enfant d'âge préscolaire et les facteurs qui influencent sa réussite éducative.julie_melancon@uqar.ca 